\title{
NOTAS BRIOLOGICAS DE LA PROVINCIA DE VIZCAYA. I. ESTUDIO DE LAS COMUNIDADES DE ESFAGNOS DEL PUERTO DE URQUIOLA
}

\author{
E. FUERTES LASALA (*); M. LADERO ALVAREZ $(*) \&$ \\ C. NAVARRO ARANDA (*)
}

\section{RESUMEN}

Hemos estudiado los esfagnos y las comunidades higro-turbosas del Puerto de Urquiola (Vizcaya). Se han recolectado 10 especies de Sphagnum, de ellas, 8 especies son nuevas citas para Vizcaya.

Del estudio fitosociológico de las comunidades, se describe la subasociación Sphagnetosum crassicladi subas. nova.

\section{SUMMARY}

We have studied the sphagnum and the damp boggy comunities of the Puerto de Urquiola (Vizcaya). We have collected 10 species of Sphagnum, from these, 8 species are new records for Vizcaya. From the syntaxonomic study of the comunities, we describe for the first time Helodo-Sphagnetum Lemée 1937, Sphagnetosum crassicladi subas. nova.

\section{INTRODUCCION}

En la subida a la Peña de Amboto desde el Puerto de Urquiola, se suceden distintos sedimentos cretácicos, ya sean calizas, areniscas o conglomerados, los cuales dan origen a diferentes tipos de suelos, desde los francamente ácidos hasta los que se encuentran próximos a la neutralidad. Las abundantes precipitaciones contribuyen a lavar el suelo y como consecuencia a rebajar el $\mathrm{pH}$ del mismo.

Los antiguos robledales y hayedos que cubrían las laderas de estas sierras han desaparecido, quedando únicamente algunos testigos en el Puerto y en la base de los roquedos próximos a la cumbre, así como en ciertas depresiones favorecidas, tal sucede en el Valle de Mañaria y en la vertiente norte de la Peña de Amboto. Los antiguos bosques de las vertientes sur y oeste por encima de los $800 \mathrm{~m}$, han desaparecido, debido a la fuerte presión ejercida por el hombre desde hace varios siglos. Al producirse la deforestación las tierras pardas desaparecieron, contribuyendo a

Comunicación presentada al III Simposio Nacional de Botánica Criptogámica. Málaga, 1978

1 Depratamento de Botanicia. Facultad de Farmacia. Cnisersidad Complutense. Madrid.

(*) Departamento de Botanica. Facultad de Ciencias. Universidad Complutense. Madrid. 
ello las abundantes precipitaciones, los continuos fuegos y los descuajes con fines ganaderos. En el momento actual las laderas están cubiertas por nanobrezales atlánticos incluídos en la clase Calluno-Ulicetea Br.-Bl., Tx. 1943. Los antiguos suelos, tierras pardas forestales, han dado paso a suelos oligotrofos del tipo rankeriforme e incluso en las vaguadas a la serie de los podsoles.

Los sedimentos cretácicos alternan, presentándose las calizas duras en las cumbres y las areniscas y los conglomerados en las laderas, lo que contribuye a dar suelos con una gran variabilidad de $\mathrm{pH}$.

En las vaguadas, depresiones y cubetas como consecuencia de las abundantes fuentes y veneros, que mantienen un alto grado de encharcamiento durante todo el año, determinan la existencia de ciénagas, donde se refugian un elevado número de taxones hidrófitos con óptimo a septentrion e incluibles en las clases Scheuchzerio-Caricetea fuscae (Nordh. 1936) Tx. 1936 Oxycocco-Sphagnetea Br.-Bl. \& Tx. 1943 y Molinio-Juncetea Br.-Bl. 1947.

En estos medios ecológicos permanentemente encharcados y salvo en aquellos regatos en los que las a guas a dquieren cierta velocidad, existe un estrato muscinal representado fundamentalmente por diversas especies del género Sphagnum (Dill.) Ehrh.

En este trabajo pretendemos presentar el comportamiento ecológico y sintaxonómico de las diferentes especies de esfagnos, desde las que tapizan las cubetas más profundas hasta las que se desarrollan a la sombra del brezal atlántico de Erica tetralix $\mathrm{L}$.

\section{CATALOGO DE ESPECIES}

Para la determinación específica del género Sphagnum hemos consultado las claves y trabajos monográficos más i mportantes en este campo como son: Dismier (1927), Dixon (1924), Augier (1966), Warnstorf (1911), Braithwaite (1880), Casares Gil (1925), Schimper (1857, 1860), Cardot (1886), Smith (1978).

La nomenclatura adoptada es la Paul (1931).

Se han recolectado 10 especies de esfagnos, de ellas ocho son citas nuevas para la provincia de Vizcaya.

\section{SECCION CYMBIFOLIA}

Sphagnum cymbifolium Ehrh.,

(S. palustre L.)

Distrib. general: cosmopolita.

Está citada entre Lequeitio y Ondarroa por P. Allorge; nosotros la hemos recolectado en los suelos cenagosos y borde de turberas del Puerto de Urquiola, en comunidades de Anagallido-Juncion Br.-Bl. 1967, y más concretamente en la asociación Narthecio-Sphagnetum plumulosi Touff. 1969.

Sphagnum papillosum Lindb.

Distrib. general: Circumboreal con disyunción austral. 
Representa la primera cita para Vizcaya, estando ampliamente representado en el norte de la Península Ibérica.

Es a bundante en los suelos higroturbosos del territorio estudiado acompañado por S. plumulosum Roll var. versicolor Warnst.

En nuestra zona caracteriza las comunidades más secas de Anagallido-Juncion Br-Bl. 1967.

\section{SECCION SUBSECUNDA}

Sphagnum inundatum (Russ.) Warnst.

Distrib. general: Circumboreal.

Primera cita para Vizcaya. Elemento higrófilo que se sitúa en los medios más encharcados de la turbera y en las zonas de abundante escurrentia. En el Puerto de Urquiola se comporta como característica de la asociación Helodo-Sphagnetum Lemée 1937.

\section{Sphagnum auriculatum Schimp.}

Distrib. general: Euroatlántica.

Citada por P. Allorge en Monte Sollube, Plencia y Ermua (Vizcaya). En el Puerto de Urquiola se presenta en todas las ciénagas llanas. La consideramos caracteristica del orden Anagallido-Juncetalia Br.-Bl. 1967.

\section{Sphagnum rufescens Limpr.}

Distrib. general: Circumboreal.

Primera cita para Vizcaya. Se desarrolla en las cubetas más profundas donde la masa de agua impide la fijación de los briófitos al sustrato, es por tanto uno de los que caracterizan las turberas vibrantes al pasear sobre ellas. En nuestro territorio caracteriza la subasociación sphagnetosum crassicladii que aquí proponemos como nueva.

Sphagnum turgidulum Warnst.

Distrib. general: Circumboreal.

La consideramos primera cita para Vizcaya. En nuestra zona esta especie muestra preferencia por ocupar los medios más húmedos. Difiere de la especie precedente por el menor número de poros dispuestos en series interrumpidas en las hojas rameales. Las hojas caulinares hasta la base presentan solamente algunos poros en su cara externa. Se presenta tanto en la a sociación Helodo-Sphagnetum Lemeé 1937 como en las menos encharcadas de Narthecio-Sphagnetum plumulosi Touff. 1969.

Sphagnum crassicladum Warnst.

(S. turgidum Röll)

Distrib. general: Circumboreal.

La consideramos como primera cita para Vizcaya. Ocupa áreas muy restringidas como son las cubetas profundas tapizadas por estagnos gruesos, constituyendo junto con S. rufescens Limpr. las características principales de la subasociación sphagnetosum crassicladii. 
Esta especie tiene el mismo aspecto y en zonas más septentrionales el mismo habitat que S. obesum Warnst., difiere de este por la presencia de poros en la cara interna de las hojas rameales y caulinares.

\section{SECCION ACUTIFOLIA}

Sphagnum acutifolium (Ehrh.) Warnst.

(S. nemorosum Scop.)

Distrib. general: Circumboreal con disyunción austral.

Primera cita para Vizcaya. Forma un tapiz casi continuo en las zonas más secas que incluso permiten una desecación temporal. Tiene su óptimo en los brezales atlánticos de Erica tetralix. Siguiendo a Touffet le consideramos característica de la asociación Tetralico-Sphagnetum.

Sphagnum quinquefarium (Lindb.) Warnst. var. versicolor Warnst.

Distrib. general: Circumboreal orófila.

Primera cita para Vizcaya. Elemento propio de las ciénagas altas de Scheuchzerio-Caricetea, en nuestro territorio, la hemos observado en los bordes de cubetas profundas dentro de la asociación Helodo-Sphagnetum Lemeé 1937.

Sphagnum plumulosum Röll var versicolor Warnst.

Distrib. general: Circumboreal subatlántica.

Es sin duda alguna el esfagno con mayor representación en el Puerto de Urquiola. Forma extens os tapices en 1 as tres comunidades que aquí comentamos. Primera cita para Vizcaya.

\section{FITOSOCIOLOGIA}

Como señala Fedoroff (1966), las turberas que se establecen en las regiones de clima atlántico no tienen una característica propia pero modelan generalmente el sustrato. No aparecen superficies convexas, elevadas y rodeadas periféricamente por regueros de agua. Desde el punto de vista florístico, estas landas se pueden incluir dentro del tipo de las turberas con flora atlántica tal como las definió Chouard (1931).

La fisonomía de la vegetación está netamente diferenciada en nuestro territorio en los siguientes biotopos:

a) Cubetas profundas tapizadas casi exclusivamente por esfagnos de gran robustez, completamente inmersos en el agua, de la que sólo afloran a la superficie los capítulos apicales. Se reconocen estos biotopos por su vibración al pisar sobre ellos. Son por tanto los auténticos tremedales.

b) Zonas de moderada inclinación del suelo, donde las aguas sobrantes de las cubetas fluyen muy lentamente a lo largo de la ladera. Existe ya un suelo con cierto espesor de turba, que en la base de la ladera forma una capa más gruesa. Los suelos son cenagosos e hirperoligotrofos, a los que distinguimos por la permanencia de las huellas del ganado que pastorea por los alrededores. Estas s on s egún Von 
Post (1925), las turberas soligénicas cuya extensión en el Puerto de Urquiola está en relación con la desaparición progresiva del bosque.

c) Zonas más secas, susceptible de una corta desecación a finales de verano y donde Ericaxtetralix L. entra como elemento más visible junto con algún otro nanofanerófito. Es aquí donde los brezales euatlánticos tienen su óptimo.

El estudio de las comunidades vegetales ha sido efectuado siguiendo el método de Zurich-Montpellier, siéndonos de gran utilidad para definir e identificar las diferentes a sociaciones, a menudo no siempre bien delimitadas y donde la identificación de las diferentes especies de esfagnos es muy difícil realizar sobre el terreno.

En las páginas siguientes trataremos de la composición florística, fisonomía, dinamismo y afinidades ecológicas de estos biotopos.

La posición sintaxonómica de la vegetación que crece sobre los tremedales y ciénagas no está totalmente definida, al menos en el límite meridional de suárea, donde se pone en contacto con numerosos elementos de medios parcialmente inundados durante ciertas épocas del año. Si bien en las zonas circumboreales las comunidades hidrofitas quedan perfectamente delimitadas como señala Osvald (1923, 1925) y Br.-Bl. (1967), no ocurre lo mismo al disminuiren latitud en la zona atlántica, donde el clima suave impide la presencia de determinados taxones nórdicos y por el contrario se observa la presencia de otros mas meridionales. Como ejemplo de lo anteriormente manifestado podemos señalar la ausencia casi total de ele mentos boreo-al pinos de la clase Scheuchzerio-Carice tea fuscae (Nordh. 1936) Tx. 1936 o de la euatlántica Oxycocco-Sphagnetea Br.-Bl. et Tx. 1943 en los tremedales y ciénagas del País Vasco.

Como señala Braun-Blanquet (1967), las zonas higroturbosas del País Vasco quedan cubiertas a parte del tapiz de esfagnos por un grupo de taxones atlánticos meridionales que se extienden desde el sur de Irlanda y Gran Bretaña hasta el norte de España y Portugal, a este área podemos añadir los numerosos tremedales que se extienden desde las sierras zamoranas hasta los Montes de Toledo, en la mitad meridional de España.

Al estudiar la composición florística de estas comunidades, hemos podido observar que los elementos de Scheuchzerio-Caricetea fuscae quedan reducidos a Rbynchospora alba (L.) Vahl., Carex flava L., Ranunculus flammula L., Eleocharis uniglumis (Link) Schultz, Veronica scutellat a L., Eriophorum angustifolium Honck, mientras faltan todas las especies típicas de las ciénagas nórdicas, fundamentalmente formadas por cárices y algunas otras como: Potentilla palustris (L.) Scop., Menyanthes trifoliata L., etc., que solo se encuentran en el interior de la Península en el piso subalpino.

Para otros botánicos, estos tremedales deben ser incluídos en la clase Oxycocco-Shagnetea Br.-Bl. et Tx. 1943 entre los que se encuentra Bellot $(1951,1966)$ Rivas Goday (1964) y más concretamente en la alianza Ericion tetralicis Schiwck. 1935. Si bien en el noroeste de España el carácter atlántico es mayor que en el País Vasco, tanto en uno como en otro fal tan los elementos característicos de los brezales nórdicos tales como: Vaccinium oxycoccos L., Andromeda polifolia L., Erica mackaii Hooker (solo en Galicia), Eriophorum vaginatum L., Odontoschisma sphagni (Dicks.) Dum. Pleurozia purpurea (Lightf.) Lindb., Sphagnum magellanicum Brid., Polytrichum strictum Banks., etc.

Por todo lo anteriormente expuesto, creemos que estas formaciones higrófitas deben ser incluídas como señala Braun-Blanquet (1967) en la clase Molinio-Juncetea Br.-Bl. 1947 y más concretamente en el orden Anagallido-Juncetalia Br.-Bl. 
1967. El mejor aval de lo anteriormente expuesto es el conjunto de plantas que caracterizan este orden y que se encuentran en los inventarios levantados por nosotros. Como elementos de estas comunidades destacamos: Carex laevigata Sm., Juncus bulbosus L., Narthecium ossifragum (L.) Huds., Hypericum elodes L., Carum verticillatum (L.) Koch, Anagallis tenella (L.) Murr. Pinguicula lusitanica L., Pedicularis sil- . vatica L., Wablenbergia bederacea Rchb., Scutellaria minor (L.) Huds., etc.

Dentro del orden Anagallido-Juncetalia se encuentra la alianza Anagallido-Juncion Br. Bl. 1967 a la cual pertenecen las asociaciones que comentaremos posteriormente.

\section{As. Helodo-Sphagnetum Lemeé 1937}

La vegetación muscinal está formada por los esfagnos acuáticos, semiacuáticos o higrófilos pertenecientes a la Sección Subsecunda Schlieph., representados en nuestra zona por S. crassicladum, S. rufescens, S. turgidulum y S. inundatum.

En relación a otros briófitos, si bien no nos hemos dedicado especialmente a ellos, cabe señalar la presencia de Aulacomnium palustre, Acrocladium cuspidatum y Campylium stellatum.

La asociación Helodo-Sphagnetum se sitúa en las cubetas profundas formando cinturones de vegetación, donde hemos podido distinguir dos zonas: una interna constantemente inundada, donde aparecen en exclusiva los esfagnos robustos $S$. crassicladum y $S$. rufescens, que podría ser señalada como un estado inicial, donde los estagnos a cidifican las aguas y colman poco a poco las cubetas. La profundidad de las aguas impide la implantación de las plantas superiores, por lo que consideramos que podría corresponder a una facies un poco especial y netamente empobrecida, donde creemos ver la subasociación sphagnetosum crassicladii subas. nov. Como inventario syntipus de esta subasociación hemos elegido el n. ${ }^{\circ} 9 \mathrm{~b}$.

$\mathrm{La}$ asociación tipo forma parte de un cinturón más externo, donde se instalan las plantas superiores correspondientes a los otros inventarios de la tabla n. ${ }^{\circ} 1$.

En las zonas de desagüe de la turbera, donde el agua fluye con cierta rapidez desaparecen los esfagnos y surge la vegetación de Littorelletea Br.-Bl. et Tx. 1943 representada por un fragmento de la asociación Potamogeton oblongus-Hypericum elodes (Allorge 1925) Br.-Bl. et Tx. 1952.

La asociación Helodo-Sphagnetum fue descrita en parte por P. Allorge (1925) del Macizo de Multonne y posteriormente redefinida por Lemeé (1937). También coincide en parte con la asociación Anagallido-Juncetum bulbosii Br. Bl. 1967, de la que se diferencia principalmente en la delimitaciones de área al estudiar los medios ecológicos.

En cuanto al dinamismo de la comunidad, pudimos observar que la asociación Helodo-Sphagnetum da paso en las zonas ligeramente inclinadas a una turbera con agua fluente, en cuyo biotopo se instala las as. Narthecio-Sphagnetum plumulosi Touff. 1969; .al hacerse la desecación mayor, el medio es invadido por distintos brezos, apareciendo entonces la as. Tetralico-Sphagnetum acutifolii Touff. 1969.

As. Narthecio-Sphagnetum plumulosi Touff. 1969

(Tetralico-Sphagnetum narthecietosum Lemeé 1937; Tetralico-Narthecietum Br. Bl. 1967 p.p.; Narthecio-Sphagnetum euatlanticum Duvigneau 1949 p.p.)

Se sitúa en suelos con moderada inclinación y abundante turba. Está caracterizada porel dominio en el estrato herbáceo de Narthecium ossifragum L. Esta planta, 
TABLA 1

HELODO-SPHAGNETUM LEMEE 1937

\begin{tabular}{|c|c|c|c|c|c|c|c|c|c|}
\hline Cobertura $\% \ldots$ & 100 & 100 & 100 & 100 & 100 & 100 & 100 & 100 & 100 \\
\hline Area $\mathrm{m}^{2} \ldots \ldots \ldots \ldots \ldots \ldots \ldots \ldots \ldots \ldots \ldots \ldots \ldots \ldots \ldots$ & 1 & 2 & 0 '5 & 0 '5 & $0 ’ 5$ & 0 '5 & 1 & 0 '5 & 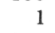 \\
\hline Altitud m. . . & 850 & 830 & 850 & 850 & 850 & 850 & 850 & 850 & 850 \\
\hline Exposición. . & $\mathrm{NE}$ & $N$ & $\mathrm{NE}$ & $\mathrm{N}$ & NW & NW & NE & NE & NE \\
\hline 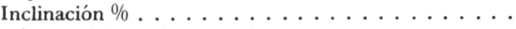 & 0 & 5 & 7 & 0 & 5. & 0 & 5 & 10 & 10 \\
\hline Número de especies. . . . . . . . . . . . . & 7 & 10 & 10 & 10 & 13 & 11 & 11 & 7 & \\
\hline Número de inventario $\ldots \ldots \ldots \ldots \ldots \ldots$ & $9 \mathrm{~b}$ & $6 a$ & $2 b$ & $4 b$ & $5 \mathrm{~b}$ & $1 \mathrm{~b}$ & $8 \mathrm{~b}$ & $6 b$ & \\
\hline
\end{tabular}

Características de asociación:

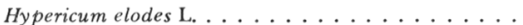

Potamogeton polygonifolius Pourr.. . . . . . . . . .

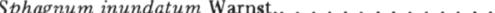

Características de subasociación:

Sphagnum crassicladum Warnst.. . . . . . . . 4 4:4

Sphagnum rufescens Limpr.

Características de alianza y orden (Anagallido-Juncion

Br.-Bl. 1967 y Anagallido-Juncetalia Br.-BL. 1967):

Juncus bulbosus L. . . . . . . . . . . . . . . . . . . . . . . . . . .

A nagallis tenella $\mathrm{L} . \ldots \ldots \ldots \ldots$

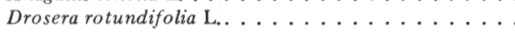

Narthecium ossifragum Huds. . . . . . . . . . . . . . . . . . . . . . . .

Scutellaria minor (L.) Huds. . . . . . . . . . . . . . . .

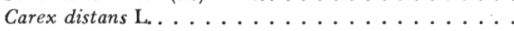

Pinguicula grandiflora Lamk.

Pedicularis sylvatica iamk.

Dactylorrhyza maculata (L.) Soó ssp. elodes (Griseb.)

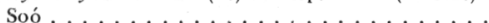

Scorzonera humilis $\mathrm{L}$

Sphagnum turgidulum Warnst. . . . . . . . . . . . . . . .

Sphagnum plumulosum Röll. . . . . . . . . . . . .

\begin{tabular}{|c|c|c|c|c|c|c|c|c|}
\hline $2: 2$ & $1: 1$ & $1: 1$ & $1: 1$ & $1: 1$ & $1: 1$ & +2 & - & . \\
\hline & . & $2: 2$ & +2 & $3: 3$ & + & $3: 3$ & $1: 1$ & $1: 1$ \\
\hline+ & $1: 1$ & $1: 1$ & . & + & $1: 1$ & + & . & $1: 1$ \\
\hline $3: 3$ & $5: 5$ & $3: 3$ & . & $\cdot$ & . & $\cdot$ & . & $1: 1$ \\
\hline . & . & . & . & $1: 1$ & . & $1: 1$ & . & • \\
\hline . & . & . & $\therefore$ & $1: 1$ & $1: 1$ & . & 1 & . \\
\hline . & & . & $1: 1$ & $i$ & . & . & + & . \\
\hline . & + & . & . & + & . & . & . & . \\
\hline • & . & • & - & + & $\therefore$ & . & . & ${ }^{\circ}$ \\
\hline & $2: 2$ & . & . & . & $1: 1$ & . & . & . \\
\hline & . & $2: 2$ & $\therefore$ & $\therefore$ & $\therefore$ & $\therefore$ & . & . \\
\hline & . & . & $2: 2$ & $2: 2$ & $4: 4$ & $5: 5$ & . & . \\
\hline
\end{tabular}

Características de clase (Molinio-Juncetea $\mathrm{Br}$-B1. 1947)

Potentilla erecta (L.) Rauschel . . . . . . . . . . . . . . .

Luzula multiflora L. . . . . . . . . . . . . . . .

Molinia coerulea Moench.

Compañeras:

Carex echinata Murr . . . . . . . . . . . . . . . . . .

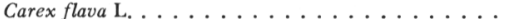

Ranunculus flammula . . . . . . . . . . . . .

Veronica scutellata $\mathrm{L} . \ldots \ldots \ldots \ldots \ldots$

Erica tetralix L.

Agrostis setacea

Rhynchospora alba Vahl.

Acrocladium cuspidatum Lindb. . . . . . . . . . . .

Campylium stellatum (Hedw.) Lang. \& Jens.

$\begin{array}{ccccccccc}+ & 2: 2 & 2: 2 & . & 4: 4 & 1: 1 & 5: 5 & 1: 1 & + \\ & + & + & 1: 1 & 1: 1 & . & 1: 1 & 1: 1 & + \\ . & . & . & 1: 1 & . & . & . & 3: 3 & 1: 1 \\ . & + & 1: 1 & . & . & . & . & . & . \\ + & + & . & . & . & . & 1: 1 & . & . \\ . & + & . & . & . & . & . & . & . \\ . & . & . & . & . & . & . & . & . \\ . & . & . & 1: 1 & . & . & . & . & . \\ . & . & . & 2: 2 & . & . & . & . & .\end{array}$

Inventario syntipus de la subasociación sphagnetosum crassicladi sub. as. nova número $9 \mathrm{~b}$ Localidad: Vizcaya, Abadiano, Puerto de Urquiola, subida a la Peña de Amboto. 
TABLA 2

NARTHECIO-SPHAGNETUM PLUMULOSII TOUFF. 1969

Cobertura $\% \ldots \ldots \ldots \ldots \ldots \ldots$

Area $\mathrm{m}^{2} \ldots \ldots \ldots \ldots \ldots$

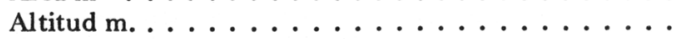

Exposición.

Inclinación $\% \ldots \ldots \ldots \ldots \ldots$

Número de especies. . . . . . . . . . . . . . . . .

Número de inventario

$\begin{array}{rrrrr}100 & 100 & 100 & 100 & 100 \\ 1 & 1 & 1 & 3 & 3 \\ 830 & 830 & 830 & 830 & 850 \\ \mathrm{~N} & \mathrm{NE} & \mathrm{NW} & \mathrm{N} & \mathrm{NE} \\ 5 & 10 & 5 & 10 & 5 \\ 13 & 10 & 14 & 12 & 7 \\ 3 \mathrm{a} & 8 \mathrm{a} & 5 \mathrm{a} & 4 \mathrm{a} & 10 \mathrm{~b}\end{array}$

Características de la asociación:

Narthecium ossifragum Huds . . . . . . . . . .

Sphagnum plumulosum Röll. . . . . . . . . . .

$4: 4$

$4: 4 \quad 1: 1 \quad 1: 1 \quad 1: 1$

Sphagnum cymbifolium ................ 1:1

3: 3

$1: 1 \quad 1: 1$

Sphagnum auriculatum.

Características de alianza y orden (Anagallido-Juncion

Br.-B1. 1967 y Anagallido-Juncetalia Br.-Bl. 1967):

Drosera rotundifolia L................ 1:1

$1: 1 \quad 2: 2$

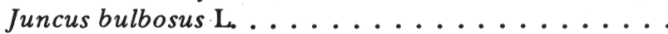

$1: 2$

$1: 1$

Eleocharis multicaulis $\mathrm{Sm}$.

$1: 1$

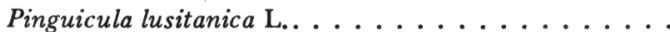

Scorzonera humilis $\mathrm{L}$.

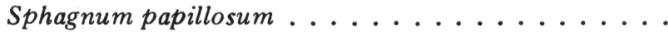

Sphagnum turgidulum Warnst.

Características de clase (Molinio-Juncetea Br.-Bl. 1947):

Potentilla erecta (L.) Rauschel ............

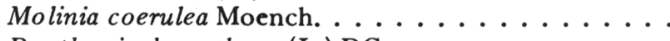

Danthonia decumbens (L.) DC

Juncus squarrosis $\mathbf{L}$

$\begin{array}{cccc}1: 1 & + & 2: 2 & 1: 1 \\ 1: 1 & 1: 1 & 1: 1 & . \\ . & +2 & 1: 1 & . \\ . & . & 1: 1 & .\end{array}$

Compañeras:

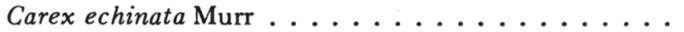

$\begin{array}{ccccc}1: 2 & 1: 1 & 2: 2 & 1: 1 & . \\ 1: 1 & . & 1: 1 & . & . \\ 1: 2 & . & 1: 1 & 3: 3 & 4: 4 \\ 1: 2 & 1: 1 & . & . & 1: 1 \\ . & + & 1: 1 & . & . \\ . & . & +2 & . & . \\ . & . & . & 1: 1 & 2: 2\end{array}$

Localidad: Vizcaya, Abadiano, Puerto de Urquiola, subida a la Peña de Amboto. 
TABLA 3

TETRALICO-SPHAGNETUM ACUTIFOLII TOUFF. 1969

Cobertura $\% \ldots \ldots \ldots \ldots \ldots \ldots \ldots \ldots \ldots$. . . . . . 100100

Area $\mathrm{m}^{2} \ldots \ldots \ldots \ldots \ldots \ldots \ldots \ldots \ldots \ldots{ }_{2}{ }_{1}$

Altitud m. . . . . . . . . . . . . . . . 830830

Exposición................. NW $\mathrm{N}$

Inclinación $\% \ldots \ldots \ldots \ldots \ldots \ldots \ldots \ldots \ldots \ldots$

Número de especies. . . . . . . . . . . . . . . . 1414

Número de inventario ................. 11a 2a

Características de asociación:

Erica tetralix L. . . . . . . . . . . . . . 2 2:2 1:2

Sphagnum acutifolium. . . . . . . . . . . . 2:2 $2: 2$

Callunas vulgaris Salisb. . . . . . . . . . . . . . 1:1 1:1

Ulex minor Roth.. . . . . . . . . . . . . . . 1:1 +1

Características de alianza y orden (Anagallido-Juncion $\mathrm{Br}$.-B1. 1967 y Anagallido-Juncetalia Br.-Bl. 1967):

Juncus bulbosus L. . . . . . . . . . . . . . . 1:1

Scorzonera humilis L. . . . . . . . . . . . . 1:1 1:1

Drosera rotundifolia L.. ............. + +

Sphagnum papillosum . . . . . . . . . . . 2:2 $\quad$ 1:1

Sphagnum plumulosum Röll. . . . . . . . . . . . . 1:1

Características de clase (Molinio-Juncetea Br.-Bl. 1947):

Potentilla erecta (L.) Rauschel. . . . . . . . . . . . 1:1 +1

Molinia coerulea Moench. . . . . . . . . . . . . . 1:1 1:1

Danthonia decumbens (L.) DC . . . . . . . . . . . 1:1 .

Compañeras:

Carex echinata Murr . . . . . . . . . . . . . 1:1 1:1

Carex flava L. . . . . . . . . . . . . . . . 1:1 1:1

Eriophorum angustifolium Roth. ........... . +

Festuca rubra L.. . . . . . . . . . . . . . . 1:1 1:1

Localidad: Vizcaya, Abadiano, Puerto de Urquiola, subida a la Peña de Amboto. 
se extiende a mpliamente en las ciénagas y tremedales del Puerto de Urquiola, cuyo estrato muscinal está formado por S. plumulosum, S. cymbifolium, S. auriculatum. Entre las especies superiores se presentan algunos ejemplares de Ericatetralix como elementos de transición a la asociación más seca de Tetralico-Sphagnetum acutifolii Touff. 1969.

En los pequeños abombamientos de esfagnos, Drosera rotundifolia L. se encuentra en condiciones óptimas de desarrollo, así como Pinguicula lusitanica L. que es menos frecuente.

La composición florística de la asociación se representa en la tabla n. ${ }^{\circ} 2$.

La presencia de $S$. plumulosum indica el óptimo del Narthecio-Sphagnetum plumulosi y la existencia de $S$. papillosum señala una facies más seca, que marca el tránsito hacia biotopos húmedos, donde como ya habíamos señalado antes, Ericatetralix L. y los esfagnos higrófilos inician su presencia.

Esta a sociación fue descrita por P. Allorge (1924) al comentar la landa turbosa de Erica tetralix. Posteriormente Lemée (1937) la denominó como Tetralico-Sphagnetum narthecietosum que corresponde a la facies de Narthecium ossifragum L. en la landa de Erica tetralix. Se asemeja también a la asociación Tetralico-Narthecietum descrito por Braun-Blanquet (1967) para las landas del País Vasco, aunque alguno de los inventarios de la tabla presentada por el autor, podrían incluirse dentro de Narthecio-Sphagnetum plumulosi, consideramos que el área que toma Braun Blanquet es más a mplia, englobando tanto a esta asociación como a la Tetralico-Sphagnetum acutifolia Touft. 1969.

\section{As. Tetralico-Sphagnetum acutifolii Touff. 1969}

Representan las zonas más secas de la turberas, suceptibles durante cortos períodos de una desecación casi total. Esta asociación es difícil de delimitar, viene caracterizada por la presencia de $S$. acutifolium acompañada a veces de $S$. papillosum. La vegetación superior está representada por la abundancia de Ericatetralix L., Calluna vulgaris L., y Molinia coerulea Moench.

La composición florística de la asociación se expresa en la tabla n. ${ }^{\circ} 3$.

Esta comunidad se instala en las landas a media pendiente, presentando abundantes brezos y brecinas y pequeños abombamientos de esfagnos, quedando surcada por pequeños arroyuelos.

En resumen, hemos distinguido en este trabajo tres asociaciones, que teniendo en común un estrato muscinal constituido por esfagnos, estos tienen un distinto comportamiento fitosociológico.

\section{BIBLIOGRAFIA}

ALLORGE, P. -1924- Etudes sur la tlore et la végátation de l'Ouest de la France. Bull. Mayenne Sc.: 77-88.

ALLORGE, P. -1925- Remarques sur quelques associations végétales du Massif de Multonne. Bull. Mayenne Si.: 27-71.

AUGIER, J. -1966- Flore des Bryophyles. Edit. P. Lechevalier. París.

BELLOT, F. -1951- Sinopsis de la vegetación de Galicia. Anal. Inst. Bot. A. J. Cazanilles: X (1): 389-444. Madrid.

BELLOT, F. -1966- La vegetación de Galicia. I. Caracteristicas de la vegetación y flora de Galicia. Anal. Inst. Bot. A.J. Cavanilles XXIV: 13-306. Madrid. 
BRAITHWAITE, R. -1880- The Sphagnaceae or peat-mosses of Europa and North America. London.

BRAUN-BLANQUET, J. -1947- Les groupements végétaux supérieurs de la France. Montpellier.

BRAUN-BLANQUET, J. -1967-Vegetationsskizen aus des baskeland mit Ausblicken auf das weitere Ibero-Atlantikum. Vegetatio 13: 117-147.

BRAUN-BLANQUET, J. \& TUXEN, R. -1943- Ubersicht der hoheren vegetationseheiten Mittele uropas. S.I.G.M.A., 84, Montpellier.

BRAUN-BLANQUET, J. \& TUXEN, R. -1952- Irische Ptlanzengesellschatten. Veroff. Geobot. Inst. Rübel in Zürich, 25. S.I.G.M.A. 117.

CARDOT, J. -1886- Les Sphaignes d'Europa. Revision critique des espéces et étude de leur variations. Bull. Soc. Roy. Bot. Belgique, 25: 136-158.

CASARES Gil, A. -1925- Los esfagnales de la Península Ibérica. Mem. Soc. Esp. Hist. Nat., 13: $1-81$.

DISMIER, G. -1927-Flore des Sphaignes de France. Sep. Arch. Bot., 1-57.

DIXON, H.N. -1924- The student's handbook of Britis Mosses. Arthur Str. W. E. London.

DUVIGNEAUD, P. -1944- Aperçu phytogéographique et phytosociologique des tourbieres de l'Ardenne Luxenbourgeoise. Bull. Soc. Bot. Belgique 76: 5-10.

FEODOROFF, N. -1966-Contribution a la conaissance de l'influence du climat sur le developpement des tourbières. C. R. Soc. Biogeogr. 42: 147-175.

LEMEE, G. -1937-Recherches écologiques sur la végétation du Perche. Thése Sc. Paris, 1388.

NORDHAGEN, R. -1936-Versuch einer euen Einteilung der subalpin-alpinen Vegetation Norwegens. Bergens. Mus. Arbok. Natuvidenskapelig rekke 1. Bergen.

OSVALD, H. -1923- Die Vegetation des Hochmoores komosse. Suenska Växtsociolg. Sällsk. Handl., I. Uppsala.

PAUL, H. -1931- Sphagnales. Die Susswaser. Flora Mitteleuropas, I. Jena.

POST, L. von. -1925- Einige Aufgaben der regionalen Moorfoschung. Szer. Geol. Unders, ser. C: $19-21$.

RIVAS GODAY, S. -1964-Vegetación y flórula de la cuenca extremeña del Guadiana. Public. Excma. Diputación provincial de Badajoz. Madrid.

SCHIMPER, W.P. -1857-Me mories pour servir a l'Histoir Naturelle des Sphaignes. Me m. Aiad. Inst. Imp. France, 15: 1-97.

SCHIMPER, W.P. -1860- Sinopsis Muscorum Europaeorum. Stuttgart.

SCHWICKERATH, M. -1938- Wälder und Waldböden des Hoben Venns und seiner Randgebiete. Mitt. Fortsäirt. u. Fortwiss. Hannover.

SMITH, A. - 1978- The moss flora of Britain \& Ireland. University Press. Cambridge.

TOUFFET, J. -1969-Les sphaignes du Massif Armoricain. Recherches phytogéographiquese técologiques. Thés. Doc. Fac. Sc. Univ. de Rennes.

TUXEN, R. -1937- Die Ptlanzengesellschaften Nordwestdeutschlands. Mitt. Flor. Soz. Arbeitsgem. Nierdersachsen, 3.

WARNSTORF, C. -1911- Sphagnologia Universalis. Das Pflanzenreich. Leipzig. 